\title{
Accelerating Advances in Cancer Care Research: A Lookback at the 21st Century Cures Act in 2020
}

Leigh Gallo, JD'; Ronald S. Walters, MD, MBA, MHA²; Jeff Allen, $\mathrm{PhD}^{3}$; Jenny Ahlstrom; Clay Alspach, JD; Yelak Biru, MSc ${ }^{6}$; Alyssa Schatz, MSW ${ }^{1}$; Kara Martin, $\mathrm{MPH}^{1}$; and Robert W. Carlson, MD ${ }^{1}$

\begin{abstract}
The 21st Century Cures Act (Cures Act), signed into law in 2016, was designed to advance new therapies by modernizing clinical trials, funding research initiatives, and accelerating the development and use of health information technology. To analyze the current issues in cancer care related to the implementation and impact of the Cures Act, NCCN convened a multistakeholder working group. Participants discussed the legislation's impact on the oncology community since enactment and identified the remaining gaps and challenges as experienced by stakeholders. In June 2020, the policy recommendations of the working group were presented at the virtual NCCN Policy Summit: Accelerating Advances in Cancer Care Research: A Lookback at the 21st Century Cures Act in 2020. The summit consisted of informative discussions and a multistakeholder panel to explore the recommendations and the future of the Cures Act. This article explores identified policy recommendations from the NCCN Working Group and the NCCN Policy Summit, and analyzes opportunities to advance innovative cancer care and patient access to data.
\end{abstract}

J Natl Compr Canc Netw 2021;19(4):378-384 doi: 10.6004/jnccn.2021.7005

\footnotetext{
${ }^{1}$ National Comprehensive Cancer Network, Plymouth Meeting, Pennsylvania; ${ }^{2}$ The University of Texas MD Anderson Cancer Center, Houston, Texas; ${ }^{3}$ Friends of Cancer Research, Washington, DC; ${ }^{4}$ CrowdCare Foundation, Salt Lake City, Utah; ${ }^{5}$ Leavitt Partners, Washington, DC; and ${ }^{6}$ International Myeloma Foundation, North Hollywood, California.
}

\section{Executive Summary}

President Barack Obama signed the 21st Century Cures Act (Cures Act) into law on December 13, 2016. The purpose of the legislation was to accelerate the discovery, development, and delivery of medical innovation. It was designed to advance new therapies by modernizing clinical trials, funding research initiatives, and accelerating the development and use of health information technology (HIT). Many initiatives within the legislation greatly impacted the cancer care ecosystem. The Cures Act created the Oncology Center of Excellence (OCE) at the FDA and provided funding to the Cancer Moonshot initiative for important oncologic research. The Cures Act also aimed to increase patientfocused drug development and the utilization of patient experience data. Finally, the Cures Act contained numerous provisions that advanced interoperability of HIT and patient access to their electronic health information. To date, the Cures Act has resulted in numerous research, medical, and technological developments; however, additional action could be taken to build on this foundation and ensure long-term success.

To analyze the current issues in cancer care related to the implementation and impact of the Cures Act, NCCN convened a multistakeholder working group on March 5, 2020. The working group developed recommendations for short-term policy strategies to address identified gaps and opportunities related to cancer care research, HIT, and other related topics.

The findings and recommendations from the working group were then presented in June 2020 at the virtual NCCN Policy Summit: Accelerating Advances in Cancer Care Research: A Lookback at the 21st Century Cures Act in 2020 to present. Additionally, thought leaders shared their unique perspectives on the future of Cures Act provisions and their impact on highquality cancer care. This article explores identified

See JNCCN.org for supplemental online content. 
policy recommendations from the NCCN Working Group and the NCCN Policy Summit, and analyzes innovative opportunities to advance cancer care and patient access to data.

\section{NCCN Working Group}

On March 5, 2020, NCCN convened a working group of 18 national experts to review the impact of the Cures Act on high-quality cancer care (see supplemental eAppendix 1, available with this article at JNCCN.org). The experts represented physicians, patients and patient advocates, researchers, policy experts, HIT experts, payers, and government representatives. The group was led by co-chairs Jeff Allen, PhD, President \& CEO of Friends of Cancer Research, and Ronald S. Walters, MD, MBA, MHA, MS, Associate Head for the Institute for Cancer Care Innovation, The University of Texas MD Anderson Cancer Center. The group discussed the successes and barriers to the implementation of Cures Act provisions related to patient access to cancer care, innovative oncologic products, and patient data, as well as the next steps needed to advance patient access to high-quality cancer care. The working group ultimately developed and endorsed 7 policy recommendations related to diversity in clinical trials, communication between the FDA and CMS, the importance of clinical trial funding, real-world data (RWD), patient data, and telehealth.

\section{Identified Barriers and Facilitators of Cures Act Implementation}

The FDA, Centers for Medicare \& Medicaid Services (CMS), Office of the National Coordinator for HIT (ONC), and other government agencies have successfully implemented numerous provisions of the Cures Act. The NCCN Working Group concluded that successes include the establishment of the OCE; the Regenerative Medicine Advanced Therapy (RMAT) designation; the advancement of patient experience data and patient-focused drug development tools; tumor-agnostic drug development; and the Cancer Moonshot initiative.

In addition to direct government action, the Cures Act increased awareness and use of RWD, increased patient access to electronic health records, and increased patient-focused drug development and legislation. Passage of the Cures Act also created greater awareness around patient data issues and increased strategic planning around interoperability and data-sharing.

Despite these robust successes, the NCCN Working Group concluded that a number of oncology-specific barriers exist to comprehensive implementation of the Cures Act. Some of the challenges and barriers discussed are as follows:

- A lack of diverse participation in clinical trials and continued stigma surrounding participation.

- Inadequate and inconsistent funding for the NCI and Cancer Moonshot.

- Unaddressed logistical challenges to patient participation in clinical trials, including transportation, cost and reimbursement, and balancing participation with personal responsibilities, such as employment and childcare.

- Challenges related to patient access to their data and a lack of actionable data for patients and providers. Additionally, utility of electronic health data is limited due to a lack of standardization throughout the health system.

- Expensive and time-consuming data collection often creates an increased administrative burden on clinicians. For patients, real-time, relevant data collection is often repetitive.

\section{Table 1. Policy Recommendations}

\begin{tabular}{|c|c|}
\hline I & $\begin{array}{l}\text { Congress must provide robust, sustained, and predictable annual funding increases to guarantee continued clinical advancements and clinical trial } \\
\text { success }\end{array}$ \\
\hline II & $\begin{array}{l}\text { The Department of Health and Human Services must encourage increased interagency collaboration and communication to enhance the oncology } \\
\text { product approval process and benefit future coverage processes }\end{array}$ \\
\hline III & $\begin{array}{l}\text { The FDA should establish parameters for the use of real-world data, including patient reported outcomes, postmarket analysis, and the } \\
\text { representation of diverse population groups, to leverage the use of real-world evidence }\end{array}$ \\
\hline IV & $\begin{array}{l}\text { Future federal legislation and Department of Health and Human Services regulatory and research initiatives should encourage and incentivize } \\
\text { widespread patient participation in clinical trials to increase diverse study populations }\end{array}$ \\
\hline V & $\begin{array}{l}\text { The Department of Health and Human Services should build upon the regulations of the Office of the National Coordinator for Health Information } \\
\text { Technology to expand available standardized data elements in a manner that is actionable to the patient and the provider }\end{array}$ \\
\hline VI & $\begin{array}{l}\text { Congress and payers should promote policies and reimbursement mechanisms that support interoperability and encourage the aggregation of } \\
\text { patient data that will promote shared decision-making and increase understanding between patients, providers, and payers }\end{array}$ \\
\hline VII & $\begin{array}{l}\text { The Centers for Medicare \& Medicaid Services and private and commercial payers should maintain and build upon advancements made throughout } \\
\text { the COVID-19 pandemic to enhance patient access to telehealth services and should increase access to tumor boards to improve patient care }\end{array}$ \\
\hline
\end{tabular}


The following policy recommendations from the NCCN Working Group aim to expand the success of the Cures Act and reduce implementation barriers (Table 1).

\section{NCCN Working Group Policy Recommendations}

I. Congress should provide robust, sustained, and predictable annual funding increases to guarantee continued clinical advancements and clinical

trial success.

The Cures Act allocated $\$ 4.8$ billion in funding to the $\mathrm{NIH}$ for 4 innovative scientific initiatives: the All of Us Research Program, the Brain Research through Advancing Innovating Neurotechnologies (BRAIN) Initiative, the Cancer Moonshot initiative, and the Regenerative Medicine Innovation Project. The Cures Act specifically allocated $\$ 1.8$ billion for the Cancer Moonshot initiative over 7 years, although yearly amounts varied. Funding peaked in the fiscal year (FY) 2019, with a total of $\$ 400$ million allocated, and will be at its lowest in FY2023, with a total of $\$ 194$ million allocated. ${ }^{1}$ Since its inception, the Cancer Moonshot initiative has funded research initiatives surrounding the prevention and early detection of hereditary cancers, to develop new enabling cancer technologies, to minimize cancer treatment's debilitating side effects, and to build a national cancer data ecosystem among others. ${ }^{2}$

For continued clinical advancements, and to consistently fund future research, Congress must provide adequate, sustained funding to the Cancer Moonshot initiative, NCI, and FDA. Research conducted by NCI and the Cancer Moonshot initiative has greatly benefited patients with cancer and survivors, and the programs established at the FDA have helped facilitate the development and efficient review of new therapies. Reliable and continuous research funding, through the annual appropriations process or allocated in Cures 2.0, will guarantee that the oncology field will continue to rapidly advance and improve patient outcomes. Annual funding increases for NCI and FDA should be exempt from mandatory, congressional budget caps. Moving forward, if research is not funded for an extended time, projects that are in process would terminate and future oncologic advancements will be severely limited. Additional funding specifically allocated to the NCI for clinical trials will guarantee future innovation in cancer care. The Annual Report to the Nation on the Status of Cancer recounted that, from 2001 to 2017, cancer rates declined among all major racial and ethnic groups, across genders, and for various age groups. ${ }^{3}$ Continuous funding that is sustained and predictable will guarantee continued clinical success and will promote future advancements in cancer care research.
II. HHS should encourage increased interagency collaboration and communication to enhance the oncology product approval process and benefit future coverage processes.

The Cures Act mandated that the FDA create Intercenter Institutes that "shall develop and implement processes for coordination of activities." This led to the creation of the FDA OCE, which works to advance the development and regulation of oncology products. In 2019, the FDA approved 11 new molecular entities for oncology, granted 27 supplemental approvals for additional oncology indications or patient populations, approved 3 biosimilars, received 4 premarket approval applications, and had 4 premarket approval modifications. Since its establishment, the OCE has led the review of entirely new classes of products, including the first cancer gene therapies, and pioneered innovative approaches to drug development, such as tumor site-agnostic approvals that were based on common genetic alterations rather than the tissue of origin. The OCE coordinated work across the various medical product centers throughout the FDA, and with the cancer research community, to make these advancements possible.

Additionally, the Cures Act provides the FDA with additional authority to establish new expedited product development programs, such as the RMAT designation and the Breakthrough Devices Program. From 2017 through 2020, the FDA, through the Center for Biologics Evaluation and Research, received 129 requests for RMAT designation. In that same period, the FDA granted 47 approvals to advance regenerative gene therapies that are "intended to treat, modify, reverse, or cure a serious or life-threatening disease or condition." ${ }^{4,5}$ Once approved, researchers have increased access to the FDA, and the drug is eligible for priority review and accelerated approval. This presents an opportunity for increased front-end collaboration toward the efficient development of novel therapies.

However, opportunities for collaboration must begin earlier in the oncology product development process and span multiple agencies to maximize greater effectiveness and access. For example, early communication with other agencies, including CMS, could help ensure the prompt establishment of appropriate coverage and reimbursement policies for new products. Improved communications between FDA and CMS surrounding novel technologies that require different payment policies will facilitate patient access to innovative treatments and efficient care. Collaboration efforts would benefit patients by guaranteeing that novel products are accessible and reimbursable without delay. New therapies must be affordable and accessible to all patients. Earlier interactions between 
the FDA and CMS will ensure that reimbursement processes and requirements for innovative products are in place to prevent delays in access. Moving forward, programs should be established through which the FDA can inform CMS about forthcoming oncology product approvals and initiate processes at CMS earlier to ensure that innovative therapies are accessible and provide value to patients, payers, and providers in a timely manner.

\section{The FDA should establish parameters for use of} RWD, including patient-reported outcomes, postmarket analysis, and the representation of diverse population groups, to leverage the use of real-world evidence.

The Cures Act defines real-world evidence (RWE) as "data regarding the usage, or the potential benefits or risks, of a drug derived from sources other than randomized clinical trials." ${ }^{\prime, 7}$ In December 2018, the FDA released the Framework for FDA's RWE Program, which defined RWE as "the clinical evidence about the usage and potential benefits or risks of a medical product derived from analysis of RWD." ${ }^{8}$ The framework states that examples of RWD include data derived from "electronic health records; medical claims and billing data; data from product and disease registries; patient-generated data, including from in-home-use settings; and data gathered from other sources that can inform on health status, such as mobile devices." The Cures Act promotes the utilization of RWE for the approval of a new indication of an approved drug. This is especially important in oncology, because many cancer drugs are proven effective for multiple types of cancers and may be able to be combined to further benefit patients and improve outcomes. ${ }^{9}$

The FDA RWE framework provides an outline of appropriate metrics, paving the way for future efforts to support data collection initiatives. To further leverage the use of RWE, and to implement the framework, the FDA should develop methodological guidance and standards to facilitate the development and use of data that include diverse populations, patient-reported outcomes, and the postmarket analysis of drugs and therapies in future data collection projects. Congressional appropriations for future initiatives would guarantee an action-oriented and productive project. Recent experiences with the COVID-19 pandemic have reemphasized the importance of high-quality RWE and reiterated the value of data collection from minorities and underrepresented population groups. The FDA must implement guardrails on the new processes to guarantee long-term benefit monitoring and influence future decision-making. Although the Cures Act encourages the use of patient experience data in the drug development process, incentivizing better data collection through payment models and regulations that mandate the collection of patient-reported outcomes and the analysis of RWE will further enhance patient care.

\section{Future federal legislation and HHS regulatory} and research initiatives should encourage and incentivize widespread patient participation in clinical trials to increase diverse study populations. Clinical trials are fundamental to the success of innovative cancer therapies. Modernizing participation will further enhance cancer care. Clinical trials can offer patients access to the most current cancer care, treatment by experts, carefully monitored results tracking, and the ability to help other patients. Patients and their care providers should be educated about clinical trial benefits during the course of treatment. Additional financial and educational resources would greatly benefit participants and the future of cancer care.

Years after passage of the Cures Act, clinical trials still struggle with the successful recruitment and retention of minority patients. Although the Cures Act recognizes the need for "increased inclusion of underrepresented populations in clinical trials," it does not mandate action. ${ }^{10}$ Instead, the Cures Act encourages the National Institute on Minority Health and Health Disparities to include "ways to increase representation of underrepresented populations in clinical trials" in its 2020 to 2024 strategic plan. ${ }^{10}$ A 2019 study found that of the 230 clinical trials leading to FDA oncology drug approvals over the past decade, race was reported in only $145(63 \%)$ trials, and of those trials that did report, people of color were significantly underrepresented. ${ }^{11}$ In 2019, after 3,593 patients participated in clinical trials, 11 new oncology drugs were approved by the FDA. Of those participants, only $4 \%$ were Black or African American and 5\% were Hispanic. ${ }^{12}$ Additionally, most clinical trials are restricted to adults between ages 18 and 64 years, resulting in an underrepresentation by children and older adults.

Future legislation and Department of Health and Human Services (HHS) regulatory initiatives should continue to encourage diverse population participation in trials and provide incentives to researchers to include underrepresented populations. Legislative and regulatory initiatives must focus on directly reducing disparities within trials and must not increase the administrative burden to NCI or researchers.

Additionally, access to life-saving therapies for Medicaid enrollees requires the coverage of routine patient costs associated with participation in qualifying clinical trials. Medicaid is currently the only major insurer that does not cover routine costs, including the nonexperimental costs of treating a patient who is participating in a clinical trial, such as the cost of physician 
visits, laboratory tests, and transportation. It is imperative that payers, employers, and CMS cover the costs associated with clinical trials. Efforts such as the Clinical Treatment $\mathrm{Act}^{13}$ are essential to increase clinical trial participation.

Understanding the benefit of clinical trials is one factor that could normalize clinical trial participation. Patient navigators can play a key role in connecting patients to clinical trials. Enhanced education programs for patient navigators on accessing clinical trials can help patients overcome barriers. Combined efforts by Congress, the FDA, HHS, and the private sector will help strengthen and diversify clinical trials.

\section{The HHS should build upon the regulations of the ONC for HIT to expand available standardized data elements in a manner that is actionable to the patient and the provider.}

Many provisions in the Cures Act address interoperability, electronic health information, and data blocking. Final regulations were released in March 2020, but a continued commitment for patient access to their electronic health data are essential for future legislation and regulations. Giving patients access to their information is an important first step; however, future regulations need to mandate that the same data elements are being supported across data systems and HIT vendors. When data are presented in an accessible, easy-to-understand format, providers and patients can use information in a meaningful, productive way.

The ONC released the 21st Century Cures Act: Interoperability, Information Blocking, and the ONC HIT IT Certification Program final rule on March 9, 2020. ${ }^{14}$ Provisions within the rule will support the exchange and use of electronic health information and promote interoperability. Since the rule's release, the ONC has extended compliance dates for certain requirements. These delays are necessary to prevent increased administrative and financial burden on the healthcare ecosystem during the COVID-19 response. Moving forward, in order to not inhibit attainment of the goals of the Cures Act, provisions must be enacted as soon as reasonably feasible. Implementation of new regulations must be balanced against the challenges created by the COVID19 public health emergency.

Current datasets are limited and should be built upon to provide effective information to patients and providers. Expanding the data elements that are collected through interoperability will increase utilization and breadth. As technology advances, ONC must continue to update and provide guidance on best practices for patients, providers, and payers. By continually adapting regulations, the ONC will help guarantee that patient electronic health information is valuable to patients. Patient access to their health data will greatly benefit their well-being and improve cancer care.

\section{Congress and payers should promote policies and reimbursement mechanisms that support interoperability and encourage the aggregation of patient data that will promote shared decision-making and increase understanding between patients, providers, and payers.}

Access to personal health data empowers patients to make informed decisions about their care. Legislation and regulatory initiatives should focus on making data accessible to patients in a format that is easy to read and comprehend. Electronic medical records should provide standardized data points so that software can communicate information effectively to patients and providers. It is important to note that a key component of making data meaningful is ensuring its timeliness. The healthcare sector must move toward investment in systems that allow rapid submission of, processing of, and access to data. Additionally, patients should have the ability to access personal databases with health information that will provide them with the most personal benefit. Initiatives increasing access and aggregating data can be encouraged and enforced through reimbursement mechanisms. Interoperability support, through policymaking and reimbursement, is essential for shared decision-making.

VII. CMS and private and commercial payers should maintain and build upon advancements made throughout the COVID-19 pandemic to enhance patient access to telehealth services and should increase access to tumor boards to improve care. On November 15, 2018, CMS released the Cures Act mandated report on telehealth. The report outlines Medicare and Medicaid populations that may benefit from telehealth services, services that may be suitable for telehealth, and barriers to telehealth access. CMS concluded that "stakeholders generally agree that telehealth can bring medical care into communities with limited access to healthcare providers, reduce wait times for patients, and be more convenient than traveling to a provider's office in both rural and urban areas."15

The COVID-19 pandemic created an urgent need for expedited patient access to telehealth services. Future legislation and rulemaking should be cognizant of the innovative ways in which the healthcare system adapted to the challenges during the COVID-19 pandemic. In response to this pandemic, CMS and other payers significantly relaxed telehealth restrictions, including geographic requirements and requirements regarding patient locations (ie, patients could be seen remotely from their home rather than a specific provider office). Additionally, the HHS Office of Civil Rights 
waived penalties for HIPAA violations against healthcare providers who serve patients through everyday communication technologies, such as FaceTime or Skype. Moving forward, CMS must enhance the telehealth reimbursement model so that providers are reimbursed at an equal rate to in-person services. For example, under the public health emergency, CMS permitted audio-only equipment to furnish certain telehealth services. ${ }^{16}$ After the public health emergency, reimbursement should continue to not distinguish between video and telephone services to prevent the disenfranchisement of patients who do not have access to video technology. Increasing access to video and audio-only telehealth services will also benefit patients with visual or hearing impairments. By making this flexibility permanent, CMS and private payers will create telehealth coverage and reimbursement policy that is more equitable to all patients.

To accommodate increased demand, many providers invested in infrastructure to support telehealth activities. Given the significant investment throughout the pandemic, telehealth policies should be evaluated and considered for their benefits beyond the pandemic, particularly as remote services become more common to accommodate societal changes. Moving forward, Congress can support the expansion of telehealth services through legislation expanding coverage of telehealth services by government and commercial insurers.

Legislation and coverage policy expanding access to and reimbursement of tumor board review is particularly important. Physicians and providers can use tumor board review to increase patient access to high-quality cancer care. Virtual treatment planning between oncologists and other specialists to create a multidisciplinary approach to care greatly benefits patients with cancer. Guaranteeing long-term access to telehealth services and tumor board review is a forward-thinking solution that will improve accessibility, decrease health disparities, and increase quality of care.

\section{Moving Innovation, Patient-Centric Care, and Patient Access to Information Forward}

The NCCN Policy Summit explored and expanded on the themes of the NCCN Working Group policy recommendations. Although many policy challenges remain, there are encouraging advancements in both the public and private sectors. Discussions with Amy Abernethy, MD, PhD, Principal Deputy Commissioner Office of the Commissioner at the FDA, and Don Rucker, MD, MBA, MS, the National Coordinator for HIT at the ONC, focused on the impact of RWD, RWE, and patient access to their health data.

RWD provides invaluable insight into the effectiveness and safety of how a drug performs. As Dr. Abernethy noted at the summit, the FDA utilizes both RWD and RWE for supplemental indications, postmarketing commitments, and postmarketing requirements. She explained that the most effective dataset includes data obtained in a research setting. According to Dr. Abernethy, including both evidence streams creates a longitudinal dataset that will tell the story of the patient's journey. Moving forward, increased access to personal and aggregated patient data would improve individual outcomes and benefit oncology research.

Dr. Rucker and the ONC are working to improve patient access to their data through computer programming and regulation. Their goal is to empower patients. Dr. Rucker described Fast Healthcare Interoperability Resources (FHIR) as a standard that "defines how healthcare information can be exchanged between different computer systems regardless of how it is stored in those systems." ${ }^{17} \mathrm{He}$ explained that these standards create access to secure clinical and patient data by making health technologies, such as mobile applications and electronic health records, easier to use by incorporating medical terminologies into the application programming interface. Standardization and collaboration when creating health technologies will increase interoperability and efficiency.

Patient access to health data has improved as a direct result of the Cures Act, but many obstacles remain. Currently, patients can access their individual health data; however, in the future, correlating patient data can be used to improve clinical outcomes and patient wellbeing. Comparing personal data with those of others who have similar diagnoses can provide valuable insight and empower patients. To guarantee that data are productive, platforms must have standardized data points that are easily accessible to all users. Fragmented data points and incompatible technologies will be inefficient and ineffective for patients and providers.

Up-to-date patient data can greatly benefit oncology research throughout the product lifecycle. RWD to build upon past clinical trials can influence future study design and identify critical information gaps. Searchable data can direct researchers to qualified patients and technology can filter appropriate exclusion and inclusion criteria. When monitoring the patient, technology can show patterns of treatment and fill information gaps. Active and passive data collection during monitoring can further reduce uncertainty and increase transparency. Additional endpoints, beyond survival, can include patient experience, pain, symptoms, laboratory test results, and imaging findings. Technologies that support patient-focused drug development in the future will need to include endpoints from across the data ecosystem and the patient lifespan.

US Representatives Diana DeGette (D-CO) and Fred Upton (R-MI) continue to advocate for innovation within the health system. On April 27, 2020, they published a concept paper outlining areas of focus for Cures 2.0. ${ }^{18}$ 
Policy initiatives include improving diversity in clinical trials, increasing the use of RWD/RWE, and encouraging patient engagement in healthcare decision-making. ${ }^{19}$ Reps. DeGette and Upton both spoke at the NCCN Policy Summit, and Rep. DeGette noted that Cures 2.0 "is really an extension of the original Cures bill to try to make it even better and to refine it." As discussed at the NCCN Working Group and NCCN Policy Summit, Cures 2.0 advancements are essential to continue advancing patient-centric, innovative oncology care.

Modern technology and continued, patient-centric advancements will empower patients and create clinically meaningful outcomes. The Cures Act, and future legislation, can continue to advance innovation and provide necessary pathways for oncologic advancement.

\section{Conclusions}

The Cures Act provided valuable resources to the oncology community through direct research funding and governmental rulemaking. Since implementation, it has benefitted cancer care through the Cancer Moonshot initiative and the OCE. Patients with cancer have further benefitted from increased access to their data. However, as the NCCN Working Group concluded, additional policies are necessary to further advance the goals set forth in the Cures Act.

Ultimately, as the oncology community continues to make strides in research, patient-centric care, and patient data, stakeholders must continue to advocate for innovative solutions. Additional advocacy from a broad coalition of stakeholders is essential to enable continued access to high-quality, comprehensive cancer care. Future policy initiatives, including Cures 2.0, must continue to expand on the important progress created by the Cures Act. Embracing innovation in the future will continue to benefit the entire health ecosystem.

Submitted November 25, 2020; accepted for publication January 5, 2021.

Disclosures: Dr. Ahlstrom has reported serving on patient advocacy boards for Sanofi, Bristol Myers Squib, Janssen, and Takeda. The remaining authors have disclosed that they have no financial interests, arrangements, or affiliations with the manufacturers of any products discussed in this article or their competitors.

Funding: This work was supported by funding from Adaptive Biotechnologies (IDOEFEAE4152); Amerisource Bergen (IDOEXIAE4153); Amgen (IDOEJNAE4154); Apobiologix (IDOE4RAE4155); Astellas Pharma US (IDOEPWAE4156); AstraZeneca (IDOED2AE4157); BeiGene, Ltd. (ID0EX6AE4158); bluebird bio (ID0EKEAG4159); Dendreon Pharmaceuticals (IDOE3IAG4160); Eisai, Inc. (IDOEONAG4161); EMD Serono (IDOEASAG4162); Exelixis (IDOEUWAG4163); Foundation Medicine (IDOEI2AG4164); Genentech (ID0E16AG4165); GlaxoSmithKline (IDOEOEBG4166); Halozyme (IDOECJBG4167); Heron (IDOEUNBG4168); Incyte Corporation

(IDOEGSBG4169); Janssen Oncology (IDOEYWBG4170); Pharmaceutical Companies of Johnson \& Johnson (IDOEK2BG4171); Kite, A Gilead Company (ID0E36BG4172); Merck (IDOEPEAI4173); MorphoSys US, Inc. (ID0EDJAI4174); Regeneron Pharmaceuticals (IDOEVNAI4175); Sun Pharmaceuticals (IDOEJSAI4176); Takeda (IDOE2WAI4177); Verastem Oncology (ID0EN2AI4178); Boehringer Ingelheim (ID0E66AI4179); and Pharmacyclics, an AbbVie Company (IDOETEBI4180).

Correspondence: Leigh Gallo, JD, National Comprehensive Cancer Network, 3025 Chemical Road, Suite 100, Plymouth Meeting, PA 19462.

Email: gallo@nccn.org

\section{References}

1. National Institutes of Health. The 21st Century Cures Act. Accessed April 6, 2020. Available at: https://www.nih.gov/research-training/medical-researchinitiatives/cures

2. National Cancer Institute. Cancer Moonshot Research Initiatives Accessed April 6, 2020. Available at: https://www.cancer.gov/research/ key-initiatives/moonshot-cancer-initiative/implementation

3. National Cancer Institute. Annual Report to the Nation: Cancer Death Rates Continue to Decline. Accessed April 6, 2020. Available at: https://www.cancer. gov/news-events/press-releases/2020/annual-report-nation-2020

4. US Food and Drug Administration. Cumulative CBER Regenerative Medicine Advanced Therapy (RMAT) Designation Requests Received by Fiscal Year. Accessed April 3, 2020. Available at: https://www.fda.gov/vaccines-bloodbiologics/cellular-gene-therapy-products/cumulative-cber-regenerativemedicine-advanced-therapy-rmat-designation-requests-received-fiscal

5. 21st Century Cures Act, HR 34, 114th Cong, Sec 3033 (2016).

6. 21st Century Cures Act, HR 34, 114th Cong, Sec 505F(b) (2016).

7. 21st Century Cures Act, HR 34, 114th Cong, Sec 505F(a)-(c)(1) (2016). Accessed April 6, 2020.

8. US Food and Drug Administration. Framework for FDA's Real-World Evidence Program. Accessed April 6, 2020. Available at: https://www.fda. gov/media/120060/download

9. National Cancer Institute. Off-Label Drug Use in Cancer Treatment. Accessed April 6, 2020. Available at: https://www.cancer.gov/aboutcancer/treatment/drugs/off-label

10. 21st Century Cures Act, HR 34, 114th Cong, Sec 2044 (2016).

11. Loree JM, Anand S, Dasari A, et al. Disparity of race reporting and representation in clinical trials leading to cancer drug approvals from 2008 to 2018. JAMA Oncol 2019;5:e191870.
12. US Food and Drug Administration. 2019 Drug Trials Snapshots: Summary Report. Accessed September 15, 2020. Available at: https://www.fda. gov/media/135337/download

13. Clinical Treatment Act, HR 913, 116th Congress (2019).

14. 21st Century Cures Act: Interoperability, Information Blocking, and the ONC Health IT Certification Program. Fed Regist 2020;25642-25961. 45 CFR 170.

15. Centers for Medicare \& Medicaid Services. Information on Medicare Telehealth. Accessed November 15, 2018. Available at: https://www.cms gov/About-CMS/Agency-Information/OMH/Downloads/Information-onMedicare-Telehealth-Report.pdf?utm source=Telehealth $+{ }^{\prime \prime \prime}$ Enthusiasts\&utm_campaign $=218 \mathrm{~d} 4 \mathrm{f} 1 \mathrm{f} 30$-EMAIL_CAMPAIGN_2018_11 26_11_35\&utm_medium =email\&utm_term $=0$ _ae00b0e89a$21 \bar{d} \mathrm{~d} 4 \mathrm{f} 1 \mathrm{f} 30-353223937$

16. Centers for Medicare \& Medicaid Services. Physicians and Other Clinicians: CMS Flexibilities to Fight COVID-19. Accessed August 20, 2020 Available at: https://www.cms.gov/files/document/covid-19-physiciansand-practitioners.pdf

17. DeGette, Upton unveil next steps for 21 st Century Cures 2.0. Accessed April 27, 2020. Available at: https://degette.house.gov/media-center/press-releases/degette-upton-unveil-next-steps-for-21 st-century-cures-20

18. The Office of the National Coordinator for Health Information Technology. What is FHIR? Accessed August 21, 2019. Available at: https://www.healthit.gov/sites/default/files/2019-08/ONCFHIRFSWhatlsFHIR pdf

19. 21st Century Cures 2.0. Accessed April 27, 2020. Available at: https:// degette.house.gov/sites/degette.house.gov/files/Cures\%202.0\%20Concept\%20Paper_0.pdf

See JNCCN.org for supplemental online content. 
Supplemental online content for:

\section{Accelerating Advances in Cancer Care Research: A Lookback at the 21st Century Cures Act in 2020}

Leigh Gallo, JD; Ronald S. Walters, MD, MBA, MHA; Jeff Allen, PhD; Jenny Ahlstrom; Clay Alspach, JD;

Yelak Biru, MSc; Alyssa Schatz, MSW; Kara Martin, MPH; and Robert W. Carlson, MD

J Natl Compr Canc Netw 2021;19(4):378-384

eAppendix 1: NCCN Working Group Members 


\section{eAppendix 1. NCCN Working Group Members: NCCN Policy Summit: Accelerating Advances in Cancer Care Research: A Lookback at the 21st Century Cures Act in 2020}

Jeff Allen, PhD, Working Group Co-Chair; Friends of Cancer Research

Ronald S. Walters, MD, MBA, MHA, MS, Working Group Co-Chair; The University of Texas MD Anderson Cancer Center Jenny Ahlstrom, CrowdCare Foundation

Clay Alspach, JD, Leavitt Partners

Alan Balch, PhD, National Patient Advocate Foundation

Al B. Benson III, MD, Robert H. Lurie Comprehensive Cancer Center of Northwestern University

Yelak Biru, MSc, International Myeloma Foundation; Walmart

Henry (Joe) Henk, MSc, PhD, OptumLabs

Jamie Holloway, PhD, Breast Cancer Survivor and Molecular Science Liaison at Caris Life Sciences

Sonya Jooma, MA, NIH All of Us

Wui-Jin Koh, MD, National Comprehensive Cancer Network

Anobel Odisho, MD, MPH, UCSF Helen Diller Family Comprehensive Cancer Center

Jon Retzlaff, MBA, MPA, American Association of Cancer Research

Alyssa Schatz, MSW, National Comprehensive Cancer Network

Kent Springfield, MBA, University of Colorado

Susan Stiles, MHA, MBA, FACHE, Cerner Corporation

Diana Verrilli, McKesson

Amanda J. Walker, MD, Johns Hopkins Medicine 